\title{
The effects of nicotine in the treatment of keloids: a pilot study
}

\begin{abstract}
Background: Dermal keloid scarring after minor injuries remain a problem in genetically predisposed individuals. Simple excision results in return of keloids within $4-6$ months in more than $50 \%-80 \%$ of patients. Available treatment options, such as steroid injections and radiotherapy, are not a hundred percent effective and cannot be used in all persons. Nicotine influences wound healing by suppressing Vascular Endothelial Growth Factor (VEGF), thought to play a key role in keloid pathogenesis, as well as suppressing inflammatory processes and possibly Transforming Growth Factor Beta (TGF-Beta). The authors sought to investigate if a nicotine containing cream may influence return of keloids post excision.
\end{abstract}

Methods: A prospective observational study was performed on outpatients at the DGMAH outpatient clinic. After standard excision, a period of 48 hours was given prior to eight hourly application of a nicotine containing cream to the wound. This application continued for 9months with follow up post excision. Patients were examined regularly with Cotinine blood tests performed within 2 months after initiation of cream application. A total of 40lesionson 31 patients were excised.

Results: Of the 31 patients 5 patients were excluded. Of the remaining 26 patients there was no return of keloid scarring in 15 patients. 2patients demonstrated a return of keloids whilst 9patients healed with hypertrophic scar formation. These did not progress to keloids at conclusion of the study.

Conclusion: Nicotine containing cream may be beneficial in preventing the return of keloids post excision. A randomized control trial with longer follow-up period may be warranted.

Keywords: keloids, hypertrophic scars, nicotine, office based surgery, patients
Volume 4 Issue 6 - 2017

Toni Jay, Nat Dip Rad

Sefako Makgatho Health Sciences University, South Africa

Correspondence: Toni Jay, MBChB, Sefako Makgatho Health Sciences University, primary surgeon, Postnet suite BI45 Private Bag XI8 Lynwood ridge, Pretoria, South-Africa 0040, Gauteng, South Africa, Email drtonijay@gmail.com

Received: May 26, 2017 | Published: October 10, 2017

\section{Aim and objective of study}

To determine whether topically applied nicotine has an effect in preventing the return of keloids following excision. This would serve as a pilot study to indicate whether a beneficial effect was evident and further randomized control trials would be justified.

\section{Introduction}

\section{Background}

Dermal keloids are an ongoing problem for genetically predisposed patients. Minor injuries may result in abnormal scarring. Surgical excision results in a return of keloids in $50 \%-80 \%$ of cases, and available treatment options, namely steroid injections, radiation therapy and silicone dressings, or pressure garments are not always feasible options. These methods are not hundred percent effective, cannot be used in all persons, have side effects (for instance pain on injection), and are not effective to treat large areas. Even dermal substitutes have resulted in keloid return. ${ }^{1,2}$ The ideal treatment should be painless, have few side effects, should be useful in large areas (for instance post burn scars) and should be easy to use maximizing compliance. The cost of the intervention should render it affordable. Steroid injections are painful, cannot be used successfully in large areas or children and there are costs involved. Triamcinolone is not always available as other steroids are purchased in the primary care setting. Radiation cannot be used in large areas and various side effects are associated. It is not safe for use in the very young or fertile age groups. Pressure garments demonstrate inadequate results and compliance. Seldom is the right amount of pressure obtained and loose fitting garments are a problem. Dermal substitutes show high return rates at high cost. ${ }^{3}$ The burden of keloids is an ongoing problem. Resources of staff, drugs and dressings are utilized repeatedly with unsatisfactory results for patients and staff alike. The psychological burden on patients, especially young people with keloids is tremendous, leading to loss of adequate social functioning. This in turn may lead to loss of productivity in the school or workplace. ${ }^{4,5}$ The study sought to determine if a cream, painless and easy to apply, produced at very low cost may be the answer to this burden. Our hypothesis was that this cream may be applied to large areas post excision, leading to maintenance of normal skin texture.

No animal model exists for any keloid research. Keloids are not formed by animals; thus all research was to be performed on consenting human participants. Lastly note that there is a distinction to be made between keloids and hypertrophic scarring. Keloids involve scar tissue enlargement beyond the boundaries of the scar, whereas hypertrophic scars do not enlarge beyond the boundaries of the scar. Under light microscopy there is little appreciable difference between the two. However, keloids show more epidermal atrophy and hypereosinophilic deposits with acellular collagen. Hypertrophic scars display more neovascularization than keloids. ${ }^{5}$ Keloids demonstrate a high level epidermal expression of Vascular Endothelial Growth 
Factor (VEGF). ${ }^{6}$ This is a potent angiogenic factor. There is an altered angiogenic balance in keloids and this was proposed be a key to therapeutic intervention. Abnormal angiogenesis is due to an imbalance between proangiogenic and antiangiogenic factors and in numerous studies it was observed that VEGF levels are unregulated whilst endostatin levels were down regulated in keloidpatients. Furthermore there is an increase in sub epidermal venous vessels as well as excessive luminal bulging of the endothelium in both arterial and venous vessels. ${ }^{8}$ Studies performed in rat lungs demonstrated that nicotine given to a pregnant rat during pregnancy decreased VEGF and VEGFR-2mRNA expression in the lungs of postnatal rats. ${ }^{9}$ Studies have furthermore proven that Dexamethasone induced keloid regression through suppression of VEGF expression and keloid fibroblast proliferation. ${ }^{10}$ As mentioned steroid injections are however painful and cannot be applied to very large areas. It was hypothesized that nicotine can firstly influence keloid formation by its down regulating effects on VEGF.

Keloids show exaggerated inflammation with production of excess extracellular matrix. There is augmented neovascularization with macrophages, mast cells and others directly and indirectly involved in the activation of fibroblasts which in turn leads to production of excess extracellular matrix. ${ }^{11}$ Nicotine is known to have antiinflammatory properties and oral treatment with nicotine in a rat model showed renoprotective effects reducing renal inflammation and halting renal protein loss where the rat had proteinuria-induced renal inflammation. The density of renal macrophages and myofibroblasts were reduced by nicotine and the level of renal inflammatory markers was also reduced. The authors suggested evaluating nicotine as a potential therapeutic option for treating proteinuric kidney disease. ${ }^{12}$ A further study was performed to investigate the effect of nicotine in an experimental mouse model of injury to the skin and subsequent healing during the inflammatory phase of repair. They found a down regulation of growth factor expression in mouse skin and macrophages. ${ }^{13}$ It is thus hypothesized that nicotine can influence keloid formation by its anti-inflammatory properties, and the idea of using nicotine as therapy is not a novel idea. The benefits of nicotine in the inflammatory processes of Ulcerative colitis are also well known. Myofibroblasts generate contractile forces within wounds and are linked to the formation of hypertrophic scars. ${ }^{14}$ It has been proven that smoking alters myofibroblastic phenotype regardless of its origin..$^{15}$ Thus we have a potential third mechanism by which topical nicotine may reduce the formation of keloids. It has been proven that nicotine modulates mast cell activity and smokers suffer less from allergic type and inflammatory type diseases. Nicotine inhibits the formation of allergic asthma in brown Norway rats. ${ }^{16}$ It has been proven that mast cells are involved in angiogenesis through the release of pro-angiogenetic factors among which tryptase is one of the most active. These tryptase-positive mast cells may play a key role in keloid angiogenesis. ${ }^{17}$ The effect of nicotine on mast cell function may be another mechanism by which nicotine may alter keloid formation. In summary on histopathological evaluation keloids comprise many distinct regions with hyalinising collagen bundles, fine fibrous areas, areas of inflammation, a zone of dense regular connective tissue, nodular fibrous areas and an area of angiogenesis. There are also exaggerated phases of vascular and fibrous granulation and remodeling stages. Furthermore there is overproduction of extracellular matrix components. ${ }^{18}$ This points to the fact that there is excessive healing taking place in the keloid with dysfunctional collagen metabolism. Smoking has to date been associated with impaired wound-healing in its most simplified form ${ }^{19}$ and this effect may very well prove useful in the prevention of "exaggerated healing" in keloid scar formation. In conclusion it has been proven, not just hypothesized that there is impairment in the quality of life in a large group of patients with keloid scarring with elements of pain, pruritus and restriction of mobility being prominent. ${ }^{20}$ No animal model exists with the closest correlation being equine exuberant granulation tissue being compared to human keloids. In this study done in 2013 they concluded that the two conditions were separate entities and only keloids contained "keloidal" collagen. Humans and horses are the only mammals known to develop excessive granulation during wound healing, but there are many differences between fibroblast populations and associated collagen, making keloid formation a condition unique to humans. ${ }^{21}$ Most notable is an article published in 2013 where it was acknowledged that smokers have faster and less erythematous scar healing to non-smokers. These findings were based on the evaluation of reduction mammoplasty scars in smoking and non-smokingpatients. ${ }^{22}$

\section{Methods}

\section{Study design}

A Prospective observational study.

\section{Inclusion criteria}

Persons in whom available treatment options have failed, not been practical to employ due to area size, or where the patient refused a form of treatment. Patients had to be older than 18years.

\section{Exclusion criteria}

Patients with fibro proliferative diseases, malignancies or diagnoses other than keloids were excluded. Patients younger than 18 years or incapable of signing informed consent and smokers were excluded.

\section{Materials, apparatus and instruments}

A nicotine cream was made by mixing aqueous cream with a known concentration of nicotine. Initial concentrations of $10 \mathrm{mg}$ of nicotine were placed into $100 \mathrm{ml}$ of aqueous cream. (This is a very low concentration of nicotine, as FDA approved nicotine patches contains up to $21 \mathrm{mg}$ of nicotine applied during 24 hours, thus $0,11 \mathrm{mg}$ vs $8 \mathrm{mg}$ over 8 hours). The rationale behind choosing this dose is that, in the absence of research where nicotine cream is applied, the only reference being nicotine patches, the authors wanted to choose a dose lower than that used in standard nicotine patches. The reason for this is that the sample group consisted on non-smokers and a small dose may well have been effective in a localized area. Nicotine patches at the time of investigation was not readily available in South-Africa and could thus not be used in the pilot study. This $10 \mathrm{mg}$ of nicotine was applied for the duration of cream application per container, which was estimated to be 30 days for $100 \mathrm{ml}$. Total duration of application was 9months. To monitor the systemic absorption of nicotine, a serum cotinine tests was performed within two months of the start of application. Patients were closely monitored to ensure no side-effects developed, however unlikely with the low concentrations used. After keloid or hypertrophic scar excision a period of 48 hours was allowed prior to cream application. The reason for the 48 hour period was that the wounds were closed for this amount of time to absorb any oozing. After 48hours the dressing was removed. Application 
of the cream then commenced immediately with three times daily applications for a period of at least 9 months. The cream was not to be massaged into the area but just applied in a manner to ensure sufficient absorption. This time period was based on the prolonged inflammatory phase observed in keloid scar formation. The T1/2 of cotinine is 20 hours. It is thus the case that after 4 to 7 days post the use of a nicotine containing product the test may not be positive anymore. The cotinine test was done on a clinic visit day at about 9 am in the morning. As the cream was applied 8 hourly, the morning dose of cream would have been applied in the region of 2 to 3 hours prior to bloods being drawn. We would thus safely be in the time frame of definite detection of cotinine in the bloodstream. The initial scar or keloid was measured at time of presentation, and then post excision immediately sent for histopathological diagnosis with micrographs to establish abnormal histopathological appearance and documentation of the aforementioned. Photographs of the keloid or hypertrophic scar in situ were also taken at initial presentation for later comparison. Immediately post op photographs were taken, and at six month follow-up further photographs were taken to assess if return of keloid or hypertrophic scarring had occurred. Measurements on lesions were be done at these time periods by means of a calliper measuring device. Only one calliper was used. If further excision was needed due to recurrence at 6 months or 9 months, the specimen was sent for histopathological analysis and micrographs, and these micrographs then compared to the original micrographs for histopathological features such as abnormal collagen formation and neovascularization.

\section{Results}

40lesionsin 31patients were excised. 5Patients (7lesions) were excluded. Reasons included: 1 was breastfeeding, 2patients had lesions other than keloids, 1 patient was non-compliant and the last patient was lost to follow-up. A total of 34lesions in 26patients were thus followed up and included in the study. Of the remaining 34 lesions, 26lesions were located on 1 or both ears, 2lesions on the scalp, 2lesionson the neck, 1 lesion on the chest and 1 lesion on the mandible. 1 lesion was located on the abdomen, and 1 lesion was located on the cheek. Maximum cross-sectional diameter per lesion ranged between $1 \mathrm{~cm}$ and $10 \mathrm{~cm}$, with an average diameter of $3.2 \mathrm{~cm}$. There was no evidence of keloid return in 15 of the 26patients. 9 of the patients formed hypertrophic scars at 9month follow-up. 2patients had what appeared to be definite return of keloids by macroscopic examination. Cotinine levels measured varied from 0 to 471 . Average cotinine levels were measured at 120.7 during cream application. Of the 11patients that had a return of either hypertrophic scarring or keloids, 6patients admitted to stopping use of the cream at 6months duration. In these 6patients, 4 patients recorded seeing the first signs of scar enlargement after 1month of stopping the use of the cream. The remaining 2 of these patients could not give a temporal relationship. Of the 5patients that did not admit to cessation of the use of the cream, 1 patient had a return of hypertrophic scarring only 8 months after excision. This patient had failed previous radiotherapy and corticosteroid injections with return of keloids within 3months post excision and use of these modalities. 1patient had two lesions excised and only one of the two lesions healed with a hypertrophic scar. The other lesion remained flat and healed well. A $3^{\text {rd }}$ patient had bilateral ear keloids with hypertrophic scarring of only one of the lesions. Of all the patients only 2 patients allowed re-excision of hypertrophic scars of keloids. 1Patient had a small hypertrophic scar and the other had what appeared to be early keloid formation. Return of keloids or hypertrophic scars was only noted in 1 patient prior to $7-8$ months. This patient had a cotinine level of 0 . The remainder namely 10patients only saw a return of keloid or hypertrophic scars after 8months. Cotinine levels in thepatients where hypertrophic scar was seen ranged from 0 to 265 with an average value of $93.8 \mathrm{ng} / \mathrm{dl}$. The 2 patients who had a return of keloids as opposed to hypertrophic scarring had cotinine values of $9 \mathrm{ng} / \mathrm{ml}$ and $211 \mathrm{ng} / \mathrm{ml}$ respectively (Tables 1-3) (Figures 1) (Figure2).

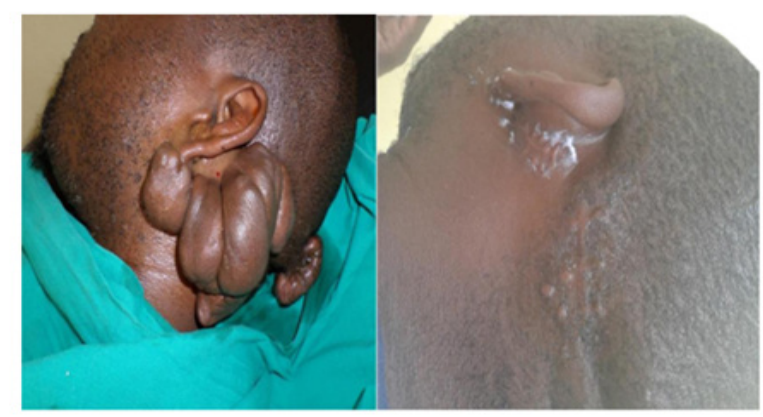

Figure I Compliant patient at 6months follow-up.

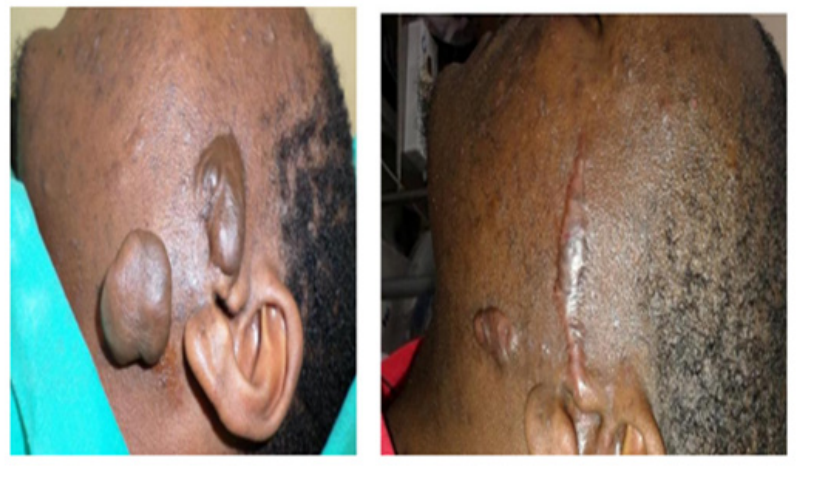

Figure 2 Non-compliant patient at 6months' follow-up. The patient ceased cream application at one-month post excision as he left the country.

Table I Location of lesions and affected body part. (Some patients had more than one lesion and some patients had lesions on both ears, but it counted as the same patient)

\begin{tabular}{ll}
\hline Location of lesion & Number of patients \\
\hline Ear & 22 (26 lesions) \\
Scalp & 2 \\
Neck & 2 \\
Mandible & 1 \\
Face & 1 \\
Chest & 1 \\
Abdomen & 1 \\
\hline
\end{tabular}


Table 2 Cotinine levels measure within 2 months of start of cream application

\begin{tabular}{|c|c|c|c|}
\hline $\begin{array}{l}\text { Patient } \\
\text { number }\end{array}$ & $\begin{array}{l}\text { Cotinine } \\
\text { value (ng/ml) }\end{array}$ & Hypertrophic scarring & Keloid return \\
\hline 1 & 471 & - & - \\
\hline 2 & 0 & Yes & \\
\hline 3 & 89 & - & - \\
\hline 4 & 161 & - & - \\
\hline 5 & 32 & Yes & \\
\hline 6 & 118 & - & - \\
\hline 7 & 265 & Yes & - \\
\hline 8 & 65 & - & - \\
\hline 9 & 0 & Yes & - \\
\hline 10 & 29 & Yes & - \\
\hline 11 & 0 & - & - \\
\hline 12 & 194 & - & - \\
\hline 13 & 177 & - & - \\
\hline 14 & 144 & Yes & - \\
\hline 15 & 9 & - & Yes \\
\hline 16 & 69 & - & - \\
\hline 17 & 211 & - & Yes \\
\hline 18 & 39 & - & - \\
\hline 19 & 391 & Yes & - \\
\hline 20 & 219 & - & - \\
\hline 21 & 327 & - & \\
\hline 22 & 40 & Yes & - \\
\hline 23 & 0 & Yes & - \\
\hline 24 & 0 & - & - \\
\hline 25 & 59 & - & - \\
\hline 26 & 0 & - & --- \\
\hline
\end{tabular}

Table 3 Size of excised lesions

\begin{tabular}{ll}
\hline Patient number & Greatest diameter of lesion excised (cm) \\
\hline 1 & 1.8 \\
2 & 2.5 \\
3 & 3.6 \\
4 & 2.8 \\
5 & 6.5 \\
6 & 3.8 \\
7 & 8.3 \\
8 & 7 \\
9 & 10 \\
10 & 2.2 \\
11 & 4.2 \\
12 & 4.4 \\
13 & 1.6 \\
14 & 3.4 \\
15 & 3.2 \\
16 & 2.7 \\
17 & 9 \\
18 & 3 \\
19 & 6.9 \\
20 & 3.7 \\
21 & 3.1 \\
22 & 1 \\
23 & 5 \\
25 & 2.8 \\
26 & 4.3 \\
\hline
\end{tabular}

\section{Discussion}

The study was designed with sole purpose was to investigate whether a randomized control trial would be feasible. When keloids are excised, 50 to 80 percent of those lesions will return within 4 to 6 months. This study showed return of keloids in 2 of the 26patients $(7,7 \%)$. The 9patients that formed hypertrophic scars (34.6\%) may progress to keloid formation. This will only become evident with longer follow up. If it is to be presumed that all 11 patients were to progress to keloids, this would leave the study with a $42.3 \%$ return rate. It can thus be assumed that the return of keloids ranged from 7.7 to $42.3 \%$. This is still below the projected $50 \%$ to $80 \%$ return rate. Also return of keloids was expected to occur at between 4-6months. Only 1 patient developed abnormal scarring during this time period. The other patients had abnormal scar formation only after 8 months. Whether or not this was associated with cessation of the use of the cream could be elucidated by the 4patients that admitted to seeing a return of scarring within 1-2months of cessation of the use of the cream. There is also a possibility that keloid return is delayed by the use of the nicotine. This seemed to be supported by the single patient that had a return of frank keloids after radiation and steroid injections within 3months, and presented with hypertrophic scarring only after 8 months of use of the cream. The study is however flawed in that the sample size is small and follow-up should ideally be for several years. A study of 3 or more years would be ideal in the case of a randomized control trial. The function of the study however, was to determine whether some effect could be elicited (Figure 3).
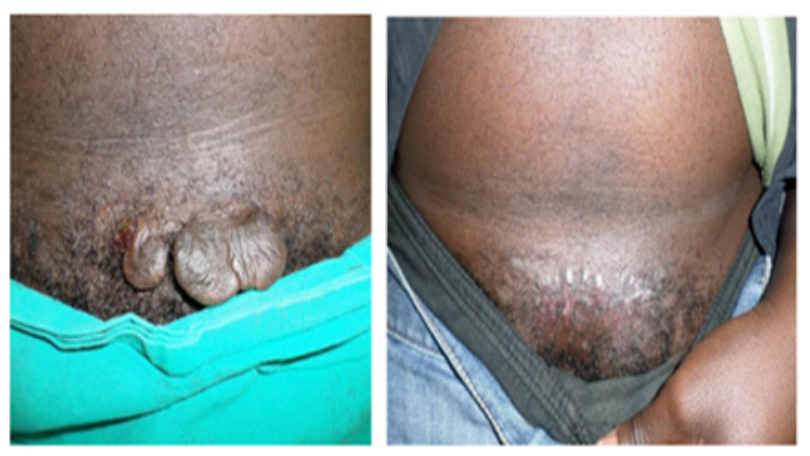

Figure $3 \mathrm{~A}$ Patient that showed no return of keloids at six months' post excision with regular use of the nicotine containing cream.

Histological confirmation was not performed on allpatients with minor hypertrophy of scars as they were satisfied and declined based on cultural reasons. Any biopsy also had the disadvantage of producing keloids or further scarring. The absence of histology in thesepatients does not influence results as the distinction between keloids and hypertrophic scarring is based on clinical judgment. The appearance of theselesionsunder light microscopy does not allow the diagnosis of either lesion to be made. Serum cotinine values differ by age and race. Significant exposure may be seen as levels more than $1 \mathrm{ng} / \mathrm{ml}$. Second hand exposure may render levels as high as 30ng/ $\mathrm{ml}$. Active smokers have cotinine levels of more than $500 \mathrm{ng} / \mathrm{ml}$. If the cream was absorbed by the users, it implied that serum cotinine levels would be detectable above the value of $30 \mathrm{ng} / \mathrm{ml}$. This was an important consideration as the cream may not have been absorbed. Average cotinine levels were $120 \mathrm{ng} / \mathrm{ml}$ in the study population which seemed to indicate that the cream was being absorbed. Average cotinine levels in the 11patients where abnormal scarring was detected 
was $93.8 \mathrm{ng} / \mathrm{dl}$ but no deductions can be made from this figure as it is merely a snapshot in time and does not contribute to our knowledge regarding patient compliance thereafter (Figure 4).patients with lower values may also not have absorbed the cream sufficiently. It is admitted that the cotinine test of the laboratory used was perhaps not of the sensitivity required for a trial, and the same was admitted by said laboratory. Be that as it may, the only deduction we may make from these measurements is that there was absorption of the cream by mostpatients, as this was a concern using aqueous cream as a vehicle. Ideally a future trial would find some correlation of dose and efficacy, and it's relation to cotinine values. Nicotine derivatives are currently being investigated as a treatment for schizophrenia and protein losing kidney disease. The cosmetic use of nicotine has not been investigated, despite support in the literature (Figure 5).

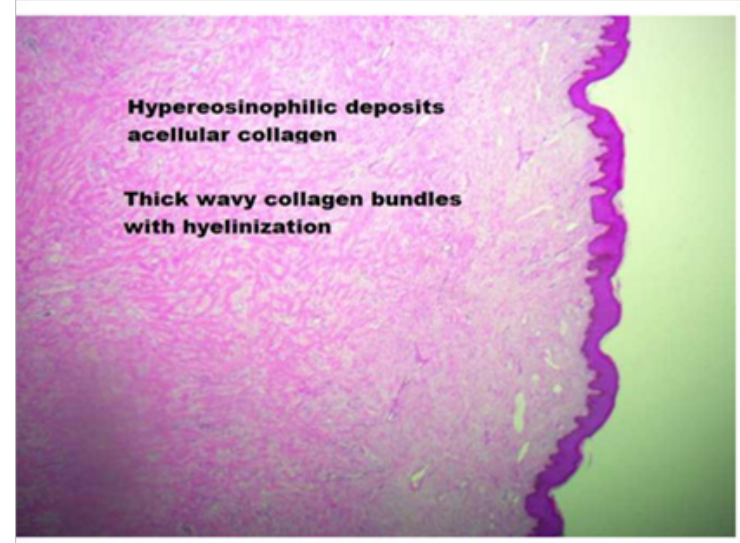

Figure 4 Normal keloid histology prior to cream application.

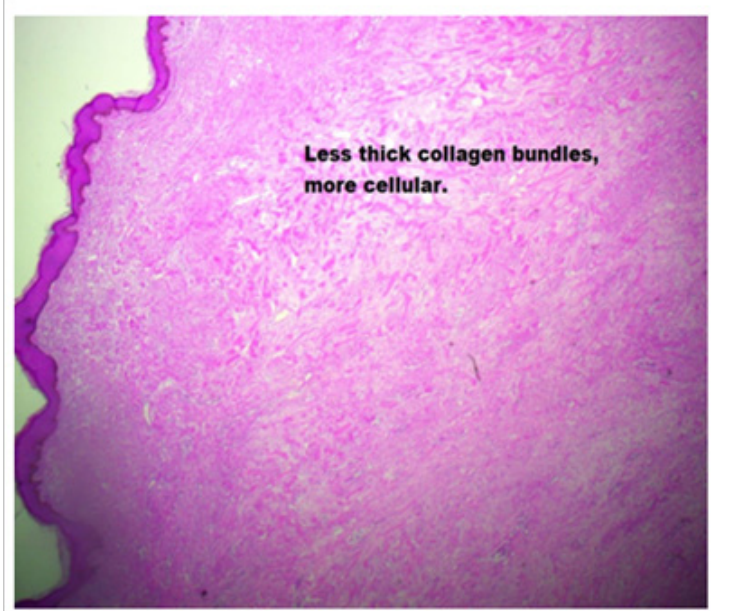

Figure 5 A Biopsy of a scar 4months after cream application. Less epidermal atrophy and less thickened collagen.

\section{Conclusion}

The literature seems to support the fact that nicotine may be useful in the prevention of keloid return. Our sample size is small, and followup times need to be several years to prove this. The nicotine does appear to have some effect on keloid return. A randomized control trial may be warranted to determine whether nicotine may be useful in the treatment of keloids. A molecular analysis of the effects on VEGF and TGF-Beta would also serve as further supporting evidence.

\section{Acknowledgements}

I declare that I have no financial or personal relationships which may have inappropriately influenced me in writing this paper.

\section{Conflict of interest}

The author declares no conflict of interest.

\section{References}

1. Meaume S, Le Pillouer-Prost A, Richert B, et al. Management of scars: updated practical guidelines and use of silicones. $J$ Eur $J$ Dermatol. 2014;24(4):435-443.

2. Song C. Hypertrophic scars and keloids in surgery: current concepts. Ann Plast Surg. 2014;1:S108-118.

3. Nyame TT, Chiang HA, Orgill DP. Clinical applications of skin substitutes. Surg Clin North Am. 2014;94(4):839-850.

4. Durani P, Bayat A. Levels of evidence for the treatment of keloid disease. J Plast Reconstr Aesthet Surg. 2008;61(1):4-17.

5. Thorne CH, Beasley RW, Aston SJ. Grabb and Smith's Plastic Surgery 6th ed. Lippincott Williams and Wilkins. 2007;243(5)893.

6. Gira AK, Brown LF, Washington CV, et al. Keloids demonstrate high-level epidermal expression of vascular endothelial growth factor. Transl Res $J$ Am Acad Dermatol. 2004;50(6):850-853.

7. Mogili NS, Krishnaswamy VR, Jayaraman M, et al. Altered angiogenic balance in keloids: a key to therapeutic intervention. Transl Res. 2011;159(3):182-189.

8. Lametschwandtner A, Staindl O. [Angioarchitecture of keloids. A scanning electron microscopy study of a corrosion specimen]. HNO. 1990;38(6):202-207.

9. Jiang JS, Chou HC, Yeh TF, et al. Maternal nicotine effects on vascular endothelial growth factor expression and morphometry in rat lungs. Early HumDev. 2012;88(7):525-529.

10. Wu WS, Wang FS, Yang KD, et al. Dexamethasone induction of keloid regression through effective suppression of VEGF expression and keloid fibroblast proliferation. J Invest Dermatol. 2006;126(6):1264-1271.

11. Van der Veer WM, Bloemen MC, et al. Potential cellular and molecular causes of hypertrophic scar formation. Burns. 2009;35(1):15-29.

12. Agarwal PK, Van den Born J, Van Goor H, et al. Renoprotective effects of long-term oral nicotine in a rat model of spontaneous proteinuria. Am J Physiol Renal Physiol. 2012;302(7):F895-904.

13. Xanthoulea S, Deliaert A, Romano A, et al. Nicotine effect on inflammatory and growth factor responses in murine cutaneous wound healing. Int Immunopharmacol. 2013;17(4):1155-1164.

14. Nedelec B, Ghahary A, Scott PG, et al. Control of wound contraction. Basic and clinical features. Hand Clin. 2000;16(2):289-302.

15. Karvonen HM, Lehtonen ST, Sormunen RT, et al. Lung cancer-associated myofibroblasts reveal distinctive ultrastructure and function. $J$ Thorac Oncol. 2014;9(5):664-674.

16. Neerad C Mishra, Jules Rir-sima-ah R, Thomas Boyd, et al. Nicotine inhibits FceRI-induced cysteinyl leukotrienes and cytokine production without affecting mast cell degranulation through Alpha7/Alpha9/ Alpha10-nicotinic receptors. J Immunol. 2010;185(1):588-596. 
17. Ammendola M, Zuccalà V, Patruno R, et al. Tryptase-positive mast cells and angiogenesis in keloids: a new possible post-surgical target for prevention. Updates Surg. 2013;65(1):53-57.

18. Bux S, Madaree A, Bux S, et al. Keloids show regional distribution of proliferative and degenerate connective tissue elements. Cells Tissues Organs. 2010;191(3):213-234.

19. Bartsch RH, Weiss G, Kästenbauer T, et al. Crucial aspects of smoking in wound healing after breast reduction surgery. J Plast Reconstr Aesthet Surg. 2007;60(9):1045-1049.
20. Bock O, Schmid-Ott G, Malewski P, et al. Quality of life of patients with keloid and hypertrophic scarring. Arch Dermatol Res. 2006;297(10):433438 .

21. Theoret CL, Olutoye OO, Parnell LK, et al. Equine exuberant granulation tissue and human keloids: a comparative histopathologic study. Vet Surg. 2013;42(7):783-789.

22. EK Deliaert, E Van den Kerckhove, Tuinder SMJS, et al. Smoking and its effect on scar healing. Eur J Plast Surg. 2012;35(6):421-424. 